УДК 004.5/316.62

05.13.10 - Управление в социальных и

экономических системах (технические науки)

ПОДХОДЫ К РАЗРАБОТКЕ

ГЕЙМИФИЦИРОВАННЫХ ПРИЛОЖЕНИЙ

Попок Леонид Евгеньевич

к.э.н., доцент, РИНЦ SPIN-код: 8450-7190

lpopok@gmail.com

Карзенкова Ольга Сергеевна

магистрант

karzenkova_o@mail.ru

Кубанский государственный аграрный университет имени И.Т. Трубилина, Краснодар,

Россия

В статье рассматриваются подходы к разработке геймифицированных приложений, правила проектирования их сценариев и принципы вовлечения пользователей в игровой процесс.

Даны практические примеры для основных этапов проектирования приложений с полной или частичной геймификацией. Сделаны выводы о целесообразности использования смешанного подхода при разработке программного обеспечения с геймификацией и даны рекомендации для разработчиков и заказчиков по повышению коммерческой эффективности геймифицированного ПО

Ключевые слова: ГЕЙМИФИКАЦИЯ, МОБИЛЬНЫЕ ПРИЛОЖЕНИЯ, МАРКЕТИНГ, ПРИНЦИПЫ РАЗРАБОТКИ, ИНФОРМАЦИОННЫЕ ТЕХНОЛОГИИ

DOI: http://dx.doi.org/10.21515/1990-4665-154-002
UDC 004.5/316.62

05.13.10 - Management in social and economic systems (technical sciences)

\section{DEVELOPMENT APPROACHES TO GAMIFIED APPLICATIONS}

Popok Leonid Evgenevich Cand.Econ.Sci., associate professor, RSCI SPIN-code: 8450-7190, 1popok@gmail.com

Karzenkova Olga Sergeevna

Master student

karzenkova_o@mail.ru

Kuban State Agrarian University, Krasnodar, Russia

The article discusses the development approaches to gamified applications, the rules for their script designing and the user involvement principles into the gameplay. The work provides practical examples for main phases of the software design with full or partial gamification. There are conclusions made about the feasibility of using mixed approach in gamified software development and there are recommendations given for developers and IT-customers to increase the commercial effectiveness of gamified software

Keywords: GAMIFICATION, MOBILE APPLICATIONS, MARKETING, DEVELOPMENT PRINCIPLES, INFORMATION TECHNOLOGY

В настоящее время геймификация наряду с другими инновационными технологиями приобрела популярность во многих сферах: в образовании, в смежных с психологией науках, в экономике и других. Одним из самых распространенных практических применений является проектирование и разработка полностью геймифицированных приложений (в том числе мобильных) или приложений с геймифицированными элементами. $\mathrm{C}$ помощью эффективного вовлекающего процесса, разработчик или заказчик сможет извлечь больше коммерческой прибыли, нежели его конкуренты, за счет привлечения 
большей аудитории, захвата ее внимания на более длительное время и т.д. Однако, чтобы разработать эффективное с точки зрения маркетинга геймифицированное программное обеспечение, необходимо целесообразно выбирать подход к разработке такой системы.

По мнению К. Вербаха, специалиста в области геймификации, в разработке геймифицированных приложений необходимо учитывать три основных правила: «путешествие» игрока, баланс и предоставление впечатлений (опыта) [1].

Правило о «путешествии» игрока предполагает необходимость воспринимать целевую аудиторию именно как игроков. Во время работы с игрофицированным приложением игрок проходит путь, сравнимый с путешествием, которое позволяет испытать игровой опыт. Для разработчика необходимо спроектировать такое путешествие цельным, структурированным и, что немаловажно, интересным, поэтому нужно уделить внимание всем элементам: началу, основной конструкции и процессу движения к цели. Таким образом, в начале необходимо помочь пользователю включиться в игровой процесс максимально быстро и просто - для этого используют всплывающие подсказки, ограничение некоторых функциональных возможностей, представляющих сложность на старте работы, и невозможность проигрыша на первых этапах. Для основной конструкции следует подобрать одну или несколько тренировочных механик, которые позволят игроку преодолеть вышеупомянутые сложности, чтобы исключить возможность запутаться или «застрять».

Следующее правило - правило баланса - в приложениях с геймификацией берет свое начало из понятия баланса в любой игре: на каждом этапе игра должна быть сбалансирована во всех элементах, чтобы оставаться интересной для каждого пользователя. 
Последнее правило из основных подразумевает, что игрофицированное приложение должно обогащать впечатления пользователей; превращать текущий процесс деятельности в более богатый и обширный опыт, не меняя сам проиесс фундаментально.

Использование рассмотренных выше правил поможет разработчику создать увлекающее и вовлекающее игрофицированное приложение, включая эмоции игрока. Именно эмоциональный компонент опыта, получаемый при победе, нахождении решений, исследования и других элементов игры, позволит создателю приложения направлять игроков к целям обоих из них без потерь мотивации пользователя.

Естественно, перед планированием и реализацией проекта необходимо определить целевую аудиторию в деталях: возраст, соотношение полов, интересы и поведение, потребности и др. К. Вербах классифицирует игроков на победителей, исследователей, социалистов и агрессоров. Поведение победителей определяется стремлением достигнуть цель, невзирая на средства и препятствия. Исследователи в первую очередь изучают окружение, стремятся до конца разобраться в представленных возможностях. Социалисты ставят во главу угла процессы взаимодействия внутри игры, для них важно коллективное выполнение заданий, создание команд. Агрессоры - это те игроки, для которых удовольствие в игре в большей степени зависит от соперничества и жесткой конкуренции [1]. Такая классификация используется для определения потребностей потенциальных пользователей и помогает выбрать используемые игровые элементы и техники.

При проектировании и непосредственном создании геймифицированных решений разработчику важно понимать направления движения развития продукта и поддерживать целенаправленность игрового процесса. Кроме того, важно понимать, какой onыm и эмоциональный отклик получит игрок во время использования 
приложения: именно вокруг пользователя должна строиться вся система. Необходимо учитывать, что элементы игры - это лишь средства формирования опыта.

Помимо рассмотренного выше игрового баланса необходимо соблюсти и баланс между аналитическим и креативным мышлением игрока: рациональность должна гармонично сочетаться с креативностью, чтобы эффективно геймифицировать тот или иной процесс (особенно, если этот процесс является рутиной). Такой подход позволяет усилить вовлеченность игрока в деятельность.

В дизайне геймифицированных приложений одним из наиболее рациональных и универсальных решений является итеративная разработка (англ. iteration - повторение) [1], подразумевающая создание программного продукта параллельно с перманентным тестированием прототипа контрольной группой и последующим анализом результатов использования. После такого анализа предыдущие этапы непосредственной разработки корректируются с учетом выявленных недостатков, давая начало новому условному циклу «планирование реализация - проверка - оценка» вплоть до получения конечного продукта. Главным среди преимуществ итеративного подхода для создания геймифицированных приложений является наличие обратной связи тестировщиков в режиме реального времени, которая позволяет оценить успешность проекта на ранних этапах разработки.

Что касается сценариев геймифицированных приложений, важную роль играют «петли активности». Различают две разновидности таких «петель» [1]. Петля вовлечения представляет собой цикличный процесс, состоящий их трех взаимосвязанных этапов (рисунок 1), данная петля действует на уровне активности пользователей. Этапы петли активности: мотивациия - призвана побудить пользователя на совершения определенного действия; собственно, действие - совершаемая 
пользователем активность, влекущая за собой явную и эффективную обратную связь; обратная связь - реакция приложения, ложащаяся в основу следующей мотивации, порождающий новый цикл.

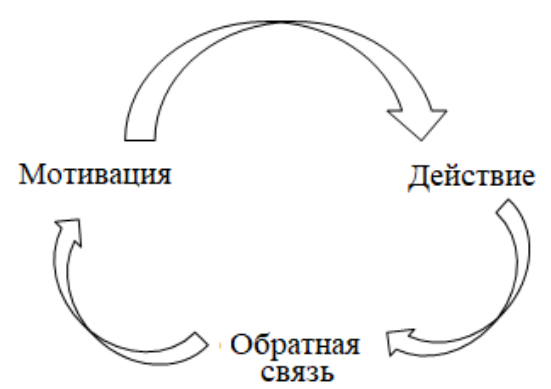

Рисунок 1 - Схема «петли вовлечения»

Второй вид петлей активности - петля прогресса. Для петли прогресса характерны два основных принципа. Во-первых, достижение цели в игре должно достигаться мелкими этапами (шагами), каждый из которых должен быть понятен игроку (явно демонстрировать цель достижения шага и дальнейшие возможности развития игры). Во-вторых, развитие игрока от начального (самого простого) уровня «новичка», к самому высокому, уровню «мастера», должно включать чередование этапов усилий и отдыха - такой своеобразный баланс позволяет сохранять и поддерживать уже установленный уровень вовлеченности игрока, не перегружая его.

Кроме вышеперечисленных подходов и принципов разработки геймифицированных приложений, существует еще два подхода к геймификации, основанные на разности в человеческом восприятии. Так, первый подход Кевин Вербах условно определяет как «деятельный», а второй - как «чувственный» [1].

Деятельный подход воспринимает понятие игры исключительно как то, что соответствуем всем формальным признакам игры, а понятие геймификации - как совокупность очков, наград и списков лидеров. В 
качестве механизма вовлечения пользователей в этом подходе используют систему стимулов и поощрений, а основной ценностью самой игрофикации для пользователя понимается удовлетворение его потребностей и желаний. Для деятельного подхода в качестве поощрения выбирается рост статуса игрока и его самооценки, а мотивацией игроков являются награды. В таком подходе первичным при создании является проработка игровых элементов и механики. Основной целью игрофикации в таком подходе является стимулирование пользователя на совершение определенного поступка.

Напротив, чувственный подход вкладывает в понятие игры всё, что воспринимается человеком как игра, независимо от формальностей, а геймификация для данного подхода - это головоломки, задачи, «вызовы» и обучение. Механизмами вовлечения пользователей, в отличие от деятельного подхода, становятся положительные впечатления и опыт, а основной ценностью геймификации - удовольствие пользователей. В качестве поощрений при чувственном подходе выступает внутренняя мотивация: новые знания, рост значимости пользователя-игрока для окружающих, a непосредственно мотивацией игроков становится демонстрация роста и прогресса, а не награды. Первичным при создании приложения в данном подходе считается разработка игровых принципов и правил, сама идея, а игровые механики и элементы являются второстепенными. Основная цель геймификации в чувственном подходе стимулирование пользователя на саморазвитие.

В качестве оптимального и целесообразного решения для большинства разработчиков геймифицированных приложений рекомендуется, помимо следования основным правилам и принципам, применение умеренного подхода, сочетающего элементы как деятельного, так и чувственного подходов в зависимости от выявленных потребностей игроков и самого разработчика. 
С точки зрения информационных технологий и разработки, настоятельно рекомендуется включать в один из начальных этапов проектирования программного обеспечения описание карты экранов (форм) приложения - схему, отражающую все экраны (формы) ПО, переходы и взаимодействия между ними, сценарные петли. Такая схема поможет выявить недостатки сценария, спроектировать полную базу данных, понять логику приложения для дальнейшей реализации. Сформированная на примере мобильного приложения «Навигационный гида по кампусу университета с элементами геймификации» карта экранов расположена на Рисунке 2. При итеративном методе разработки, который рекомендован создания для геймифицированного программного обеспечения, подразумевается пересмотр и возможное изменение (дополнение) карты навигации.

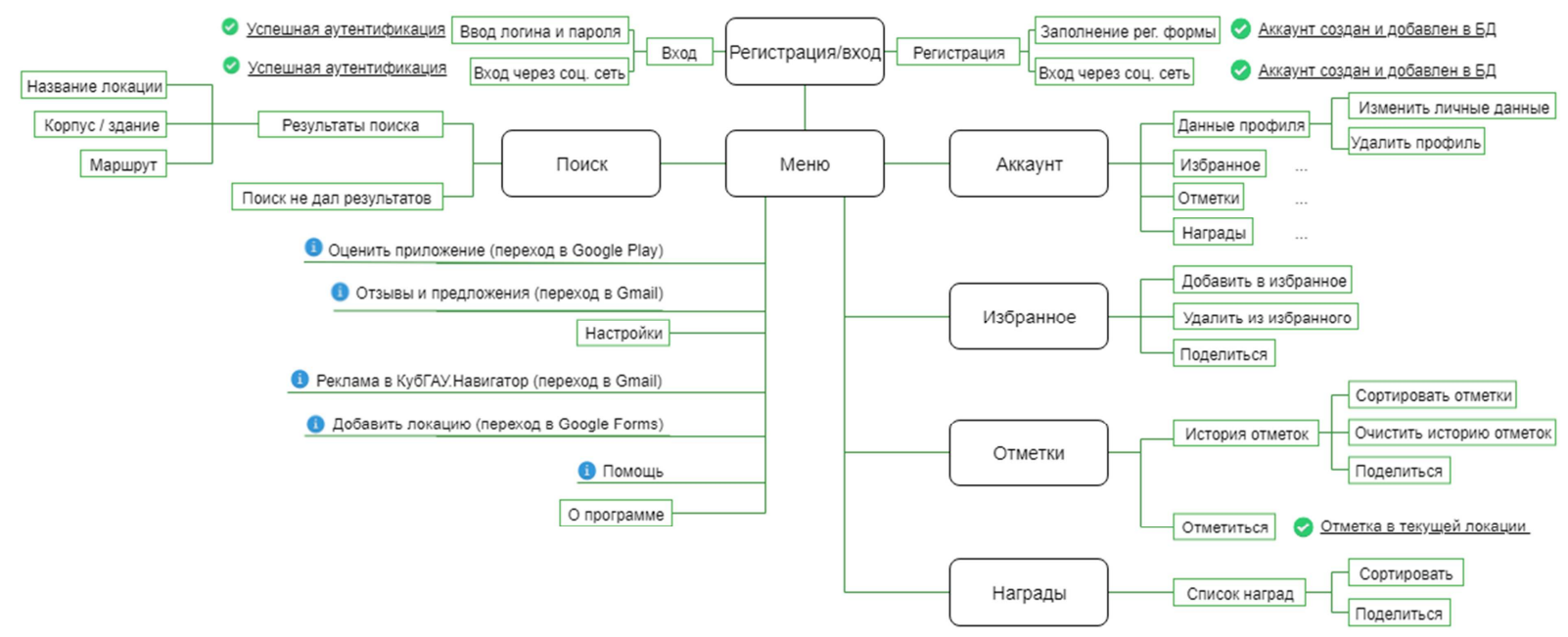

Рисунок 2 - Пример карты экранов геймифицированного мобильного приложения

При проектировании базы данных разработчику также необходимо учитывать возможность в перспективе развития программного продукта расширять и дополнять эту базу, так как вовлечение и геймификация длительные непрерывные процессы, которые со временем могут эволюционировать и требовать больше вариантов хранения данных. 
Таким образом, при разработке полностью или частично геймифицированного программного обеспечения важно найти оптимальный подход, отражающий цели использования элементов геймификации; обеспечить непрерывный процесс создания вовлекающих сценариев, созданных по приведенным правилам, с помощью итеративной разработки; учитывать психологические и другие особенности целевой аудитории. Кроме того, важно обратить внимание на этап проектирования карты навигации и базы данных приложения.

\section{Список литературы}

1. Вербах, К. Курс из 12 лекций «Геймификация» [электронный ресурс] / К. Вербах // Учебный курс Coursera - URL: https://www.coursera.org/learn/gamification и http://metaver.pbworks.com/w/page/59924682.

2. Стародубцева Е., Геймификация в компаниях [электронный ресурс] / Е. Стародубцева, Литвин П., Петрова И., Жиронкина Н.В. //Блог Работа.ру. - 2018. - URL: https://www.rabota.ru/articles/hr/game-4919.

3. Курылев, И.Н. Что такое геймификация? [электронный ресурс] / И.Н. Курылев // Игровые механики в бизнесе. - 2017. - URL: http://gamification-now.ru/wtf/.

4. Зикерманн Г. Геймификация в бизнесе: как пробиться сквозь шум и завладеть вниманием сотрудников и клиентов / Г. Зикерманн, Д.Линдер; пер. с англ. И. Айзятуловой. - М.: Манн, Иванов и Фербер, 2014. - 272 с.

5. Валерина Л.П. Геймификация в образовании [Электронный ресурс] // Историческая и социально-образовательная мысль. - URL: http://cyberleninka.ru/article/n/geymifikatsiya-vobrazovami (Дата обращения: 12.02.2017)

6. Вербах К. Вовлекай и властвуй: игровое мышление на службе бизнеса / К. Вербах, Д. Хантер. - М.: Манн, Иванов и Фербер, 2015. - 223 с.

7. Brian Burke, Gamify: How Gamification Motivates People to Do Extraordinary Things, 2014. - $192 \mathrm{c}$.

References
1. Verbah, K. Kurs iz 12 lekcij «Gejmifikaciya»/ K. Verbah // Uchebnyj kurs Coursera
- $\quad$ URL: http://metaver.pbworks.com/w/page/59924682.

2. Starodubceva E., Gejmifikaciya v kompaniyah / E. Starodubceva, Litvin P., Petrova I., ZHironkina N.V. //Blog Rabota.ru. - 2018. - URL: https://www.rabota.ru/articles/hr/game4919.

3. Kurylev, I.N. CHto takoe gejmifikaciya? / I.N. Kurylev // Igrovye mekhaniki v biznese. - 2017. - URL: http://gamification-now.ru/wtf/.

4. Zikermann G. Gejmifikaciya v biznese: kak probit'sya skvoz' shum i zavladet' vnimaniem sotrudnikov i klientov / G. Zikermann, D.Linder; per. s angl. I. Ajzyatulovoj. M.: Mann, Ivanov i Ferber, 2014. - 272 s.

5. Valerina L.P. Gejmifikaciya v obrazovanii // Istoricheskaya i social'noobrazovatel'naya mysl'. - URL: http://cyberleninka.ru/article/n/geymifikatsiya-vobrazovami 
6. Verbah K. Vovlekaj i vlastvuj: igrovoe myshlenie na sluzhbe biznesa / K. Verbah, D. Hanter. - M.: Mann, Ivanov i Ferber, 2015. - 223 s.

7. Brian Burke, Gamify: How Gamification Motivates People to Do Extraordinary Things, 2014. - 192 c. 